\title{
The vestibular implant: Opinion statement on implantation criteria for research
}

\author{
Raymond van de Berg ${ }^{\mathrm{a}, \mathrm{b}, *}$, Angel Ramos ${ }^{\mathrm{c}}$, Vincent van Rompaey ${ }^{\mathrm{d}}$, Alexandre Bisdorffe, \\ Angelica Perez-Fornos ${ }^{\mathrm{f}}$, Jay T. Rubinstein ${ }^{\mathrm{g}}$, James O. Phillips ${ }^{\mathrm{g}}$, Michael Strupp ${ }^{\mathrm{h}}$, \\ Charles C. Della Santina ${ }^{\mathrm{i}}$ and Nils Guinand ${ }^{\mathrm{f}}$ \\ ${ }^{a}$ Department of Otorhinolaryngology and Head \& Neck Surgery, Maastricht University Medical Center+, \\ Maastricht, The Netherlands \\ ${ }^{\mathrm{b}}$ Faculty of Physics, Tomsk State University, Tomsk, Russian Federation \\ ${ }^{\mathrm{c}}$ Department of Otolaryngology Head Neck Surgery, Complejo Hospitalario Universitario Insular Materno \\ Infantil de Gran Canaria, Department of Otolaryngology, Las Palmas University (ULPGC), \\ Psychoacoustic \& Equilibrium Laboratory, Las Palmas University (ULPGC) \\ ${ }^{\mathrm{d}}$ Department of Otorhinolaryngology and Head \& Neck Surgery, Antwerp University Hospital, Department \\ of Translational Neurosciences, Faculty of Medicine and Health Sciences, University of Antwerp, Belgium \\ ${ }^{\mathrm{e}}$ Clinique du Vertige, Centre Hospitalier Emile Mayrisch, Esch-sur-Alzette, Luxembourg \\ ${ }^{\mathrm{f}}$ Division of Otorhinolaryngology Head and Neck Surgery, Geneva University Hospitals and University \\ of Geneva, Geneva, Switzerland \\ g Otolaryngology-HNS, Virginia Merrill Bloedel Hearing Research Center, University of Washington, \\ Seattle, WA, USA \\ ${ }^{\mathrm{h}}$ Department of Neurology and German Center for Vertigo and Balance Disorders, Ludwig Maximilians \\ University, Munich, Germany \\ ${ }^{\mathrm{i}}$ Departments of Otolaryngology - Head \& Neck Surgery and Biomedical Engineering, Johns Hopkins \\ School of Medicine, Baltimore, MD, USA
}

\begin{abstract}
This opinion statement proposes a set of candidacy criteria for vestibular implantation of adult patients with bilateral vestibulopathy (BVP) in a research setting. The criteria include disabling chronic symptoms like postural imbalance, unsteadiness of gait and/or head movement-induced oscillopsia, combined with objective signs of reduced or absent vestibular function in both ears. These signs include abnormal test results recorded during head impulses (video head impulse test or scleral coil technique), bithermal caloric testing and rotatory chair testing (sinusoidal stimulation of $0.1 \mathrm{~Hz}$ ). Vestibular implant (VI) implantation criteria are not the same as diagnostic criteria for bilateral vestibulopathy. The major difference between VI-implantation criteria and the approved diagnostic criteria for BVP are that all included vestibular tests of semicircular canal function (head impulse test, caloric test, and rotatory chair test) need to show significant impairments of vestibular function in the implantation criteria. For this, a two-step paradigm was developed. First, at least one of the vestibular tests needs to fulfill stringent criteria, close to those for BVP. If this is applicable, then the other vestibular tests have to fulfill a second set of criteria which are less stringent than the original criteria for BVP. If the VI-implantation is intended to excite the utricle and/or saccule (otolith stimulation), responses to cervical and ocular vestibular evoked myogenic potentials must be absent in addition to the above mentioned abnormalities of semicircular canal function. Finally, requirements for safe and potentially effective stimulation should be met, including implanting patients with BVP of peripheral origin only, and assessing possible medical and psychiatric contraindications.
\end{abstract}

\footnotetext{
${ }^{*}$ Corresponding author: Raymond van de Berg. E-mail: raymond.vande.berg@mumc.nl.
} 
Keywords: Vestibular implant, vestibular prosthesis, implantation criteria, bilateral vestibulopathy, bilateral vestibular hypofunction, electrical stimulation, vestibular system, vestibulo-ocular reflex, VOR, bilateral vestibular loss, bilateral vestibular areflexia

\section{Introduction}

Bilateral vestibulopathy (BVP) is a disorder most often resulting from reduced peripheral vestibular function $[1,2]$. Patients can suffer from many disabling symptoms, like imbalance and oscillopsia (illusory visual movement of the environment) [3]. BVP leads to reduced mobility, up to 30 times higher risk of falling, significant loss of quality of life, and an increased socio-economic burden on individuals and on society [4]. The etiologies of BVP are heterogenous and vary from ototoxicity, e.g., from exposure to gentamicin or streptomycin, to genetic factors, e.g., DFNA9, CANVAS or spinocerebellar ataxia, infectious causes, e.g., meningitis, auto immunity, e.g., Cogan's syndrome, trauma, and neurodegenerative diseases $[2,5,6]$. However, in up to $51-75 \%$ of patients with BVP, the etiology remains unclear $[2-4,7]$. In some patients there may be a transition from presbyvestibulopathy to BVP due to aging or neurodegeneration [8].

A diagnosis of BVP can be made using the diagnostic criteria consensus document of the classification committee of the Bárány Society [9]. However, clinical reality shows that establishing a diagnosis of BVP is challenging in many cases [10]. Pitfalls in diagnosis of BVP include: 1) different clinical pictures with and without dizziness, postural imbalance, vertigo, or oscillopsia, 2) falsely normal clinical bedside head impulse test (HIT) results due to covert compensatory saccades, and 3) frequently normal appearing test results with rotatory chair testing, especially in the higher frequencies around $5 \mathrm{~Hz}[2,11-13]$. Finally, there is no agreement on normative data for laboratory testing of vestibular function [14]. These challenges can often lead to misdiagnoses or a delay in diagnosis, on average a delay of 33 months, up to more than 8 years [10]. Therefore, prevalence rates for BVP which vary from 28 to 81 per 100.000 people, are very likely to be an underestimation $[15,16]$. If so, neurotologists are likely to encounter more of these patients in their daily clinic than previously reported, especially in older patients.

Unfortunately, the prognosis is poor for recovery of vestibular function in patients with BVP [17]. Furthermore, symptoms often do not resolve despite participation in vestibular rehabilitation therapy and cessation of vestibular-suppressant medications. Currently available treatment options have a low likelihood of benefit, especially for unpredictable and high-frequency movements $[18,19]$.

In the last decade, multiple research groups have proposed the concept of a vestibular implant (VI) to partially restore vestibular function in patients with BVP [20-23]. The VI, which is currently still considered a research device, is in concept analogous to the cochlear implant. It captures head movements with motion sensors and processes them into electrical signals. These electrical signals are then conveyed to the vestibular nerve by electrodes that are implanted near the vestibular nerve branches that innervate the semicircular canals, i.e. the anterior, lateral and posterior canal ampullary nerves, or otolith organs, i.e. the utricular and saccular nerves, collectively called macular nerves [23-26]. Research in humans has demonstrated feasibility of the VI to partially restore vestibular function. First, it was reproducibly shown that it is possible to increase the vestibulo-ocular reflex (VOR) gain with a VI [27-30], and to influence the vestibulocollic and vestibulospinal reflexes $[31,32]$. Secondly, functional benefits were demonstrated by restoring the dynamic visual acuity in close-to-reality situations and in the high-frequency range $[33,34]$. Therefore, it was concluded that the VI could become a clinically useful device for treatment of patients with BVP, and possibly for other vestibular disorders like chronically uncompensated unilateral vestibulopathy [35]. Patients also have indicated the need for VI research; their main expectation is improvement of their overall mobility (van Stiphout et al., submitted).

Currently, one of the main current questions in the field of VI research is: Whom should be implanted for research? Until now, no uniform implantation criteria for research have been established and as a consequence, research groups have applied different criteria $[21,29,36]$. Therefore, in order to facilitate better comparison between future clinical trials regarding efficacy, safety, and other outcome measures, there is a need for establishing uniform criteria for the selection of patients appropriate for implantation of a VI.

The classification committee of the Bárány society previously described the diagnostic criteria for 
BVP, which is also called bilateral vestibular failure, -deficiency, -areflexia, -hypofunction and -loss [9]. These criteria comprise a combination of symptoms and quantitative VOR measures of the lateral semicircular canal. However, function of vertical semicircular canals and otolith organs are not included [9]. The criteria for VI-implantation should go beyond the existing criteria for BVP, in order to demonstrate significant impairment of all canals in all frequency ranges, and (on indication) the otolith organs, since:

1) VI-implantation is practically irreversible for the vestibular system: the diagnosis should therefore be verified by more than one vestibular test;

2) VI-implantation is not restricted to the lateral semicircular canals, but can also involve the other semicircular canals and the otolith organs: the function of these structures should therefore be evaluated before implantation;

3) VI-implantation can impair residual vestibular function, e.g. by surgical plugging of the semicircular canals: the VI should not damage the vestibular system more than it can help restore it.

Furthermore, VI-implantation is a unilateral procedure and may in the future not be restricted to BVP: indications might evolve to include second-side implantation or otherwise to treat chronically uncompensated unilateral vestibulopathy [35]. Although this document only focuses on BVP, other indications for VI-implantation should not be excluded from future research.

The objective of this opinion statement is to provide clear and, as far as possible, evidence-based criteria for VI-implantation in adult patients with BVP in a research setting. Since research regarding this subject is an ongoing process, it is not the aim to define criteria that are "set in stone". To the contrary, this document aims to provide a basis for the first preclinical VI-implantations, and to lay the foundation for future development of VI-implantation $[2,37-40]$.

\section{Methods}

Members from all four research groups currently investigating vestibular implantation in humans were invited to participate in this opinion statement by the first author. All four groups participated. In addition, other research collaborators and members of both the Bárány Society and Politzer Society were included. This opinion statement was developed according to the template established by the Classification Committee of the Bárány Society [41], although this is not an official document of the Bárány society. A literature search was performed and draft criteria were developed. These criteria were supported by notes and comments. An iterative process of discussion and refinement by all authors resulted in the final opinion statement.

\section{VI-implantation criteria}

The diagnostic criteria of BVP according to the Bárány Society were modified and extended, since they are a necessary but not sufficient prerequisite. Therefore, the following more precise criteria are recommended as provisional criteria for the selection of patients appropriate for implantation with a $\mathrm{VI}^{1}$. To be eligible for VI-implantation, patients must meet all of the following criteria:

A. Chronic vestibular syndrome with the following disabling ${ }^{2}$ symptoms:

- Unsteadiness when walking or standing plus at least one of the following:

- Movement-induced blurred vision or oscillopsia during walking or quick head/body movements, and/or

- Worsening of unsteadiness in darkness and/or on uneven ground

B. Symptoms greatest during head movement

C. Bilaterally reduced or absent angular VOR function documented by at least one of the following major criteria:

- Bilaterally pathological horizontal angular VOR gain $\leq 0.6$ and at least bilaterally one vertical angular VOR gain $<0.7$, measured by the video-HIT or scleral-coil technique $^{3}$

- Reduced caloric response (sum of bithermal max. peak SPV on each side $\leq 6 \%$ sec for $30 \mathrm{sec}$ water stimuli or $<10^{\circ} / \mathrm{sec}$ for $60 \mathrm{sec}$ water or air stimuli) ${ }^{4}$

- Reduced horizontal angular VOR gain $\leq 0.1$ upon sinusoidal stimulation on a rotatory chair $\left(0.1 \mathrm{~Hz}, \mathrm{Vmax}=50^{\circ} / \mathrm{sec}\right)$ and a phase lead $>68^{\circ}$ (time constant $<5 \mathrm{sec}$ ) 
C'. Obligatory only in case of implantation of otolith structures: Bilaterally absent cVEMP and oVEMP responses ${ }^{5}$

D. In case only one or two criteria from $\mathrm{C}$ are matched (and also criterion C' is matched in case of otolith stimulation), the remaining test(s) should comply with the following minor criteria:

- Bilaterally pathological VOR gains of at least two semicircular canals $<0.7$, measured by the video-HIT or scleral-coil technique

- Reduced caloric response (sum of bithermal max. peak SPV on each side $<10^{\circ} / \mathrm{sec}$ for water and air stimuli of $\geq 30 \mathrm{sec}$ )

- Reduced horizontal angular VOR gain $<0.2$ upon sinusoidal stimulation on a rotatory chair $\left(0.1 \mathrm{~Hz}, \mathrm{Vmax}=50^{\circ}\right.$ / sec)

E. Symptoms are not better accounted for by another disease

F. Fitting the additional requirements relevant to initial preclinical trials

- Age 18 years and above

- BVP results most likely from a peripheral origin $^{6}$

- Vestibular function and symptoms are unlikely to significantly improve, according to the duration of symptoms and clinicians' estimations ${ }^{7}$

- Patent vestibular end-organ and intact vestibular nerve ${ }^{8}$

- Ability to use the device and follow a personalized rehabilitation program ${ }^{9}$

- Ability to undergo the surgery ${ }^{10}$

G. No current psychological or psychiatric disorder that could significantly interfere with use or evaluation of the VI ${ }^{11}$

\section{Notes}

1. It is advised to become familiar with the notes and comments of the diagnostic criteria for BVP established by the Bárány Society [9] because patients meeting diagnostic criteria for BVP might also meet these candidacy criteria for vestibular implantation.

2. Disabling is considered as "interfering with activities of daily living". It is up to the patient and clinician to decide whether symptoms are disabling in each specific case. A Dizziness
Handicap Inventory total score of $>30$ would be preferred [42].

3. vHIT should be performed and analyzed by a trained examiner, using a validated vHIT device and taking precautions to minimize vHIT goggle slip with respect to the skull, which is common and typically results in artifacts that influence vHIT VOR gain. VOR gain is the ratio between angular eye velocity and angular head velocity. For these implantation criteria, two options are possible to calculate the VOR gain: 1) the ratio of the area under the curve of angular eye velocity over time, divided by the area under the curve of angular head velocity over time, or; 2) angular eye velocity at a fixed time (typically around $60 \mathrm{~ms}$ after onset of the impulse), divided by angular head velocity at the same time [9]. It is advised to calculate the VOR-gain of each canal using a minimum of seven artifactfree impulses. Head impulses should have a peak acceleration of at least $1000^{\circ} / \mathrm{sec}^{2}$ [11].

4. The caloric test should preferably be performed using cold and warm $\left(30^{\circ}\right.$ and $44^{\circ}$ respectively) irrigations of water, lasting for $\geq 30$ seconds, with a total volume of at least $250 \mathrm{ml}$. Alternatively, warm and cool air insufflation (8 liters/min) can be used instead of water. Air caloric tests should only be performed with a $60 \mathrm{sec}$ stimulus to be able to comply with criterion C. Precautions should be used to ensure that VOR responses to a prior stimulus are not falsely recorded as responses to a subsequent stimulus due to persistence of the previous response. Therefore, techniques designed to minimize persistence of caloric effects from one stimulus trial that could confound measurements during a subsequent trial, such as interposing a stimulus interval of five minutes between successive monothermal irrigations, should be used [11].

5. Criterion C' only applies when otolith implantation is considered. If only semicircular canal implantation is considered, VEMPs do not have to be absent in the candidate ear. However, relative function of the left and right utricle and saccule as quantified by VEMP responses, should be considered along with pre-operative semicircular canal and cochlear function when deciding whether and which side to implant.

6. A disorder of peripheral origin is defined by having an etiology localized to the inner ear (e.g. hair cell loss or dysfunction due to gentamicin 
ototoxicity, a genetic abnormality, infection, ischemia, trauma or other labyrinthine injury). No persistent central vestibular involvement can be present, e.g., no tumors of the vestibular nerve, no brainstem lesions, and no cerebellar ataxia. An idiopathic BVP without any central vestibular signs on physical examination including ocular motor testing and with a normal MRI of the brain and internal auditory canals, can be regarded as "most likely from a peripheral origin." Disorders with clear signs of structural nerve pathology, e.g., vestibular schwannoma or after vestibular neurectomy, and disorders with indications that the vestibular nerves might not be functioning properly, e.g., after radiotherapy of the cerebellopontine angle, should not be considered for VI-implantation.

7. The clinician should estimate that it is very unlikely vestibular function will recover to values higher than the criteria mentioned above, and that vestibular symptoms will significantly improve. In cases that do not require early implantation (see "Comments - Timing of implantation and rehabilitation"), it is preferred to have a "wait and see period" of at least six months from onset of symptoms, including an adequate vestibular rehabilitation program with the patient off all vestibular suppressant medications for at least three months. After that, it is up to the clinician to estimate whether vestibular implantation can be considered. If there is a delay in diagnosis of BVP, and a patient has already experienced symptoms typical of BVP for more than 6 months [10], vestibular implantation can be considered without an additional "wait and see period" as long as adequate treatment, e.g., rehabilitation off vestibular suppressants, has already been provided. In cases that require early implantation (e.g. meningitis with concern for impending labyrinthitis ossificans), it is up to the clinician to estimate whether vestibular function and symptoms are likely to recover or not. If the probability is low, vestibular implantation can be considered.

8. Prior to intralabyrinthine electrode insertion, the structures to be implanted (semicircular canals and/or otolith organs) should be determined to be patent for surgical insertion as determined by high resolution temporal bone CT. In conditions with an increased risk of a non-patent labyrinth, e.g. meningitis with con- cern for development of labyrinthitis ossificans, patency should be checked with MRI and/or CT. Patients without a patent labyrinth are not yet considered for intralabyrinthine electrode insertion. There should be no clear signs of structural nerve pathology or indications that the nerve could not be functioning properly (see note 6 ).

9. Factors for successful use of a VI system and accomplishment of a personal rehabilitation program include, among others: ability to understand and use the system, ability to tolerate the external device and implant, e.g. no allergy for the components of the device, ability to tolerate repeated activation and deactivation of the device, ability to fulfill the rehabilitation program with respect to physical condition and logistic factors, e.g. travel distance, willingness to avoid vestibular suppressant medications that can interfere with central nervous system compensation after sudden onset of asymmetry in vestibular input from the two ears.

10. The whole vestibular implant team should agree on the operability of the patient. This includes considering co-morbidities, e.g. American Society of Anesthesiologists Physical Status Classification System [43], and the status of the middle ear on the side to be implanted, e.g. no chronic otitis media.

11. If a psychological or psychiatric disorder, e.g., depression, anxiety disorder, borderline personality disorder, is present and considered likely to hinder thorough evaluation and treatment of the VI recipient according to the clinician, VI-implantation should be avoided. Persistent Postural Perceptual Dizziness (PPPD) is not considered an exclusion criterion, nor is a history of psychological or psychiatric disorders that are not currently present or are judged not likely to relapse during the research trial.

\section{Comments}

\subsection{Vestibular implantation criteria differ from bilateral vestibulopathy criteria}

In its current forms, vestibular implantation surgery can irreversibly impair any residual vestibular function. Since a vestibular test is never infallible, e.g. the caloric test results can be subject to anatomical variabilities leading to false positive outcomes [11], a two-step paradigm for vestibular testing was imple- 
mented. This implies that one abnormal vestibular test is not enough to confirm implantation eligibility: a double confirmation from the other two vestibular tests is needed. In contrast to BVP diagnostic criteria, all vestibular tests mentioned above are suggested to screen for implantation eligibility. However, not all patients suffering from severe BVP who might be candidates for vestibular implantation show consistently low vestibular test results on all vestibular tests. For example, a subject might have a caloric response of $\leq 6^{\circ} / \mathrm{sec}$ but an angular vHIT VOR gain of $>0.6$ instead of $\leq 0.6$ [42]. Therefore, for the confirmation step, criteria for vestibular test results are less strict (see point $\mathrm{D}$ of the criteria). Due to this double confirmation, the vestibular test results under point $C$ were changed from " $<$ " to " $\leq$ ". Whether all these values are still low enough to prevent clinically relevant damage of useful sensory function by vestibular implantation, should still be investigated.

Criteria in point $\mathrm{F}$ were included to increase the likelihood that VI-implantation will be safe and effective.

\subsection{Disabling symptoms}

No questionnaires are available currently that are specifically designed to determine the level of disability in BVP patients. Therefore, it was decided to defer to clinician expertise and opinion regarding the disability due to BVP in each specific case. A total score on the Dizziness Handicap Inventory of $>30$ would be preferred, since this could show at least moderate handicap [44, 45]. It was decided not to make this score obligatory since in a cohort of 90 patients with bilateral vestibulopathy, more than $40 \%$ of the patients scored below 31, while still a subset of them reported significant complaints [42]. This could imply that the Dizziness Handicap Inventory might not be the best tool to quantify the handicap in bilateral vestibulopathy patients. However, it could still help guide some clinicians in their judgment.

\subsection{Types of surgery and implantation criteria}

For vestibular implantation, two types of surgery are possible: intralabyrinthine, i.e., fenestrating the semicircular canals and/or vestibulum and inserting the electrodes, and extralabyrinthine, i.e., not intentionally opening the semicircular canals and/or vestibulum and trying to place the electrodes directly on the nerves close to the semicircular canals or the internal auditory canal [46]. The intralabyrinthine approach modifies the biomechanical properties of the canals and can lead to loss of the residual inner ear function including hearing loss. Theoretically, the extralabyrinthine approach might reduce this risk. However, the full risks of the extralabyrinthine approach are not yet fully determined, and the facial nerve is exposed to a higher risk with the extralabyrinthine approach [47]. Therefore, it was decided to consider both techniques as being associated with a risk of inducing vestibular hypofunction.

Tests of otolith function were not included in the diagnostic criteria for bilateral vestibulopathy. Since implantation of otolith structures [23] is almost certain to cause otolith hypofunction due to mechanical disruption of the membranous labyrinth during implantation, oVEMPS and cVEMPS were included in the implantation criteria when the otolith structures are being considered for implantation (see C').

\subsection{Video head impulse testing}

Based on previous findings in BVP patients, it was chosen to set the angular VOR gain of the vertical semicircular canals and the gain of the lateral semicircular canal in those patients whose caloric test or rotatory chair test already fit the implantation criteria to $<0.7$ [42]. This value might be subject to change, depending on new insights. Only one vertical semicircular canal on each side needs to fulfill the criterion of an angular VOR gain $<0.7$, since it was shown that selective sparing of a single semicircular canal is possible [48] while a clinically relevant BVP is still present, making the patient probably eligible for implantation. It should be noted that artifacts frequently occur [49] when using the Video Head Impulse Test to assess the lateral and especially the vertical semicircular canals. Therefore, test results that are noisy, distorted by artifacts, or otherwise unreliable, should not be included in the analysis [2].

Non-quantitative head impulse testing (HIT), i.e., a physical examination without a means of high speed eye movement recording, was not included in the implantation criteria, due to its limited sensitivity and specificity in the setting of covert corrective saccades [12]. Bedside HIT also is not able to quantify precisely the effect of the vestibular implant after implantation, in contrast to vHIT or scleral coil oculography.

The suppression head impulse paradigm (SHIMP) was not included in the criteria, since vHIT has already been shown to be effective in demonstrat- 
ing the vestibular deficit. The clinical relevance of SHIMPs in BVP should still be determined [50].

\subsection{The caloric test}

Since not all laboratories are able to use water irrigations, e.g., due to regulations, it was decided to also include the air caloric test in the VI-implantation criteria. When using the air caloric test, it is advised to irrigate at 8 liters/min for at least 60 seconds to be able to comply with criterion $\mathrm{C}$, since air might be a less strong stimulus than water. This paradigm is advised based on the experience with the air caloric test in patients following VI-implantation [29]. In case of a $60 \mathrm{sec}$ stimulus (water or air), $<10^{\circ} / \mathrm{sec}$ is used as a criterion rather than $\leq 6 \% \mathrm{sec}$ because stimulation for an additional $30 \mathrm{sec}$ beyond the initial $30 \mathrm{sec}$ of stimulation is very likely to increase the magnitude of the response.

Caloric test values for implantation are defined in point $\mathrm{D}$ as the sum of bithermal caloric irrigations $<10^{\circ} / \mathrm{sec}$ on each side using irrigations for $\geq 30 \mathrm{sec}-$ onds. This criterion is based on previous reports in which patients with a sum of all bithermal irrigations $<20 \%$ sec still reported significant imbalance and/or oscillopsia [2, 39]. This might make these patients candidates for vestibular implantation. However, these values might still be subject to change, depending on new insights. In both criteria $C$ and $\mathrm{D}$, water and air stimuli have the same cut-off value when irrigating for $60 \mathrm{sec}$ (criterion C) and $\geq 30 \mathrm{sec}$ (criterion D). The committee is aware that these different stimuli probably do not lead to equal results. However, experience with caloric test results as an inclusion criterion for vestibular implantation only exists with $30 \mathrm{sec}$ water stimuli and $60 \mathrm{sec}$ air stimuli. In order to facilitate centers who might use $60 \mathrm{sec}$ water stimuli, this stimulation paradigm was added to the criteria although specific values for vestibular implantation when using $60 \mathrm{sec}$ water stimuli are not available.

Ice water caloric irrigations are not required in the implantation criteria since a sum of bithermal max. peak SPV on each side $\leq 6 \%$ sec for $\geq 30 \mathrm{sec}$ water stimuli or $<10^{\circ} / \mathrm{sec}$ for $60 \mathrm{sec}$ stimuli, was judged to be adequate as a major criterion (point $\mathrm{C}$ of criteria) for vestibular implantation.

\subsection{Rotatory chair testing}

The criterion for horizontal angular VOR gain measured with sinusoidal stimulation on a rotatory chair is defined in point $\mathrm{D}$ as $<0.2$. This value is higher than the one used in the diagnostic criteria for BVP. It has been shown that patients with BVP with a horizontal VOR gain $\geq 0.1$ at a stimulus frequency of $0.1 \mathrm{~Hz}$ still experienced significant imbalance and/or oscillopsia, making them possible candidates for vestibular implantation [2]. The upper value of $<0.2$ is based on expert opinion only, and should still be investigated further in the future. For very low VOR responses, the calculation of VOR gain, and especially of VOR phase and VOR time constants may be unreliable. Results should therefore be evaluated critically and interpreted with caution [2].

\subsection{Vestibular evoked myogenic potentials}

It is preferred to test cVEMP and oVEMP responses before and after vestibular implantation. VEMP responses must be compared to normative values established for the system used by the particular laboratory [51]. In case of implantation of otolith structures, cVEMP and oVEMP responses should be absent bilaterally. Although the otolith system can probably have a gradual decline in function and does not work as an "on-off" system [52], VEMPS are not currently clinically suited to detect subtle changes in otolith function or define "minimal residual otolith function". It was decided therefore that otolith implantation should only be performed in patients with bilaterally absent VEMP responses and not take into account other parameters, e.g. increase of thresholds. More specifically, both cVEMP and oVEMP responses should be absent, since otolith implantation might interfere with the function of both otolith organs. With absent cVEMP and oVEMP responses, it is expected that the benefit of vestibular implantation will be higher than the drawbacks of the potential iatrogenically induced vestibular hypofunction due to vestibular implantation of the otolith endorgans. This criterion is subject to change, depending on new insights. Note that in case of otolith endorgan implantation, VEMPs are used in addition to the other criteria; that is, the other criteria should also be fulfilled. These patients should also have bilaterally impaired semicircular canal function and, therefore, patients suffering from so-called "dissociated bilateral vestibulopathy" are currently not candidates for VI-implantation [53].

VEMP responses do not need to be absent when only implanting the semicircular canals. Many symp- 
tomatic patients with BVP can have preserved otolith responses [37, 54-56]. The presence of VEMP responses indicates that on an aggregate basis some otolithic signal is present, but it does not demonstrate useful otolith function. Furthermore, correct implantation of semicircular canals and electrical stimulation does not necessarily lead to destruction of the otolith organs [57]. Nevertheless, the committee strongly advises to always measure VEMP responses before and after vestibular implantation, to investigate the influence of vestibular implantation on otolith structures and their responses.

\subsection{Implantation criteria include only adults}

At this moment, evidence regarding the effects of BVP in the pediatric population is considered to be too scarce to consider implanting the pediatric population. It is advised by the committee to first investigate this subject further in adults, and extend this knowledge to the development of outcome measures for vestibular implantation specifically tailored to the pediatric population.

\subsection{Implanting only BVP of peripheral origin}

The VI replaces the vestibular organ by capturing head motion and sending electrical signals to the vestibular nerves. A VI-implantation attempts to serve as an artificial replacement of the peripheral vestibular organ and it does not replace the whole vestibular nerve or central vestibular system. Only few human implantations have been performed until now $[21,23,29,36]$ and the influence of deficits of the vestibular nerve and central vestibular system on performance of the VI have not been studied yet. However, it is expected that with current techniques, the VI will not effectively treat a deficit of the vestibular nerve or the central vestibular system. Currently, it is therefore preferred to implant a patient only when symptoms mainly result from an inner ear deficit. In the future, it should be investigated whether BVP not only or not mainly resulting from an inner ear deficit, can be treated with a VI.

\subsection{Timing of implantation and rehabilitation}

The prognosis of BVP is poor in most cases [11]. Most patients do not show significant recovery from their vestibular function loss. In addition, six months after a vestibular nerve injury, recovery is probably complete [58]. Therefore, a "wait and see" period of at least 6 months after the onset of symptoms was chosen before considering vestibular implantation. In cases with a possible reversible etiology or that require early implantation (e.g. labyrinthitis ossificans), the "wait and see" period is tailored to the prognosis of the disorder comparable to cochlear implantation. Due to lack of sufficient tools to predict the prognosis for each specific case, it was decided to value the clinicians' expertise and opinion regarding the prognosis of recovery.

Data are not yet available on the effectiveness of VI-implantation and the influence of etiology and duration of the disease on efficacy. It is hypothesized that "dying back" of the vestibular nerve could compromise effective vestibular stimulation, although long standing vestibular losses have also shown promising results with a VI [29, 30, 35, 36]. For now, it is preferred to avoid a "wait and see" period of more than six months, if possible. Therefore, if BVP already exists for more than six months without any improvement, the "wait and see" period of six months is not applicable. The "wait and see" period of six months after onset of the disease also facilitates time for an adequate vestibular rehabilitation program. An adequate vestibular rehabilitation program should include once a week supervised rehabilitation sessions for three months, in addition to a daily home exercise program, as advised by the American Physical Therapy Association [59]. If symptoms are present for more than six months, it should be decided for each individual case whether vestibular rehabilitation is still indicated.

\subsection{Imaging}

An MRI-scan of the internal auditory canals and cerebellopontine angle is advised in the vestibular implantation work-up for two reasons: 1) to evaluate the presence or absence of vestibular nerves, and to evaluate the patency of the labyrinth in cases with an increased risk of a non-patent labyrinth (see note 8 ). Conditions with an absent vestibular nerve preclude VI-implantation on the side with the absent nerve. Conditions without a patent labyrinth, e.g., labyrinthitis ossificans, are not now considered for intralabyrinthine electrode insertion although in the future a "vestibular drill-out" might be considered; and 2) to screen for additional findings in cases without a definite etiology. Significant findings on MRI have been reported in 14\% of BVP-cases. Some of these findings might hinder vestibular implantation 
or influence the sidedness of implantation, e.g. unilateral vestibular schwannoma [2]. It is preferred to use high resolution $(<1 \mathrm{~mm}) \mathrm{T} 1$ and $\mathrm{T} 2$ images of the cerebellopontine angle, preferably with intravenous gadolinium contrast, to be able to detect extralabyrinthine and intralabyrinthine disorders, e.g., an intralabyrinthine vestibular schwannoma. A CT scan of the mastoid region is helpful for surgical planning, to assess labyrinth patency, and to rule out middle ear or mastoid disease that could prevent safe and successful vestibular implantation.

\subsection{Hearing and vestibular implantation}

Hearing status has explicitly not been incorporated in the vestibular implantation criteria. Hearing might be preserved in the acute phase after intralabyrinthine implantation, although this is not always the case [60]. The extralabyrinthine approach was developed in hopes of reducing the risk of hearing loss or creating vestibular hypofunction $[47,61]$. However, at the present time, only deaf patients have been implanted with the extralabyrinthine approach. The safety for hearing has therefore not yet been determined.

Two versions of the vestibular implant are available: the vestibular implant aimed at only restoring vestibular function and the vestibulo-cochlear implant aimed at restoring vestibular and hearing function by incorporating also a cochlear implant. Taking the available evidence into account, it was decided that in case of a vestibular implant only, it is too early to develop formal auditory criteria. Hearing status is therefore not a determining factor for implantation, although the hearing status of both ears should be taken into account. The better hearing ear should not be implanted. Furthermore, it is advised to only implant patients in whom, according to the clinician, coping mechanisms and the hearing status of the contralateral ear are sufficient to be able to successfully cope with a possible single sided deafness on the implanted side. With a vestibulo-cochlear implant, criteria for cochlear implantation in the ear to be implanted are applicable, in addition to the criteria for vestibular implantation.

\section{Acknowledgments}

The authors would like to thank Joe Furman for his critical review of the paper and his valuable suggestions regarding content and editing.

\section{References}

[1] T.C. Hain, M. Cherchi and D.A. Yacovino, Bilateral vestibular loss, Seminars in neurology 33(3) (2013), 195-203.

[2] F. Lucieer, P. Vonk, N. Guinand, R. Stokroos, H. Kingma and R. van de Berg, Bilateral Vestibular Hypofunction: Insights in Etiologies, Clinical Subtypes, and Diagnostics, Frontiers in neurology 7 (2016), 26.

[3] F. Lucieer, S. Duijn, V. Van Rompaey, A. Perez Fornos, N. Guinand and J.P. Guyot, et al. Full Spectrum of Reported Symptoms of Bilateral Vestibulopathy Needs Further Investigation-A Systematic Review, Frontiers in neurology 9 (2018), 352.

[4] D.Q. Sun, B.K. Ward, Y.R. Semenov, J.P. Carey and C.C. Della Santina, Bilateral Vestibular Deficiency: Quality of Life and Economic Implications. JAMA otolaryngologyhead \& neck surgery 2014.

[5] D.J. Szmulewicz, C.A. McLean, H.G. MacDougall, L. Roberts, E. Storey and G.M. Halmagyi, CANVAS an update: clinical presentation, investigation and management, Journal of vestibular research: equilibrium \& orientation 24(5-6) (2014), 465-474.

[6] S.P. de Varebeke, B. Termote, G. Van Camp, P.J. Govaerts, S. Schepers, T. Cox, et al. Focal sclerosis of semicircular canals with severe DFNA9 hearing impairment caused by a P51S COCH-mutation: is there a link? Otology \& neurotology : official publication of the American Otological Society, American Neurotology Society [and] European Academy of Otology and Neurotology 35(6) (2014), 1077-1086.

[7] V.C. Zingler, C. Cnyrim, K. Jahn, E. Weintz, J. Fernbacher, C. Frenzel, et al. Causative factors and epidemiology of bilateral vestibulopathy in 255 patients, Ann Neurol 61(6) (2007), 524-532.

[8] Y. Agrawal, R. Van de Berg, F. Wuyts, L. Walther, M. Magnusson, E. Oh, et al. Presbyvestibulopathy: Diagnostic criteria Consensus document of the classification committee of the Barany Society, Journal of vestibular research: equilibrium \& orientation 29(4) (2019), 161-170.

[9] M. Strupp, J. Kim, T. Murofushi, D. Straumann, J.C. Jen, S.M. Rosengren, et al. Bilateral Vestibulopathy: Diagnostic criteria. 2016

[10] M. Miffon and J.P. Guyot, Difficulties Faced by Patients Suffering from Total Bilateral Vestibular Loss, ORL $J$ Otorhinolaryngol Relat Spec 77(4) (2015), 241-247.

[11] R. van de Berg, M. van Tilburg and H. Kingma, Bilateral Vestibular Hypofunction: Challenges in Establishing the Diagnosis in Adults, ORL J Otorhinolaryngol Relat Spec 77(4) (2015), 197-218.

[12] C.W. Yip, M. Glaser, C. Frenzel, O. Bayer and M. Strupp, Comparison of the Bedside Head-Impulse Test with the Video Head-Impulse Test in a Clinical Practice Setting: A Prospective Study of 500 Outpatients, Frontiers in neurology 7 (2016), 58.

[13] G.M. Halmagyi, S.T. Aw, P.D. Cremer, I.S. Curthoys and M.J. Todd, Impulsive testing of individual semicircular canal function, Annals of the New York Academy of Sciences 942 (2001), 192-200.

[14] M. Strupp, J. Grimberg, J. Teufel, G. Laurell, H. Kingma and E. Grill, Worldwide survey on laboratory testing of vestibular function, Neurology: Clinical Practice 2019:10.1212/CPJ.0000000000000744.

[15] N. Guinand, F. Boselie, J.P. Guyot and H. Kingma, Quality of life of patients with bilateral vestibulopathy, The Annals 
of otology, rhinology, and laryngology 121(7) (2012), 471-477.

[16] B.K. Ward, Y. Agrawal, H.J. Hoffman, J.P. Carey and C.C. Della Santina, Prevalence and impact of bilateral vestibular hypofunction: results from the 2008 US National Health Interview Survey, JAMA otolaryngology- head \& neck surgery 139(8) (2013), 803-810.

[17] V.C. Zingler, E. Weintz, K. Jahn, A. Mike, D. Huppert, N. Rettinger, et al. Follow-up of vestibular function in bilateral vestibulopathy, Journal of neurology, neurosurgery, and psychiatry 79(3) (2008), 284-288.

[18] F. Porciuncula, C.C. Johnson and L.B. Glickman, The effect of vestibular rehabilitation on adults with bilateral vestibular hypofunction: a systematic review, Journal of vestibular research: equilibrium \& orientation 22(5-6) (2012), 283-298.

[19] H. Kingma, L. Felipe, M.C. Gerards, P. Gerits, N. Guinand, A. Perez-Fornos, et al. Vibrotactile feedback improves balance and mobility in patients with severe bilateral vestibular loss, Journal of neurology. 2018.

[20] C.C. Della Santina, A.A. Migliaccio and A.H. Patel, A multichannel semicircular canal neural prosthesis using electrical stimulation to restore 3 -d vestibular sensation, IEEE transactions on bio-medical engineering 54(6 Pt 1) (2007), 1016-1030.

[21] J.S. Golub, L. Ling, K. Nie, A. Nowack, S.J. Shepherd, S.M. Bierer, et al. Prosthetic implantation of the human vestibular system. Otology \& neurotology: official publication of the American Otological Society, American Neurotology Society [and] European Academy of Otology and Neurotology 35(1) (2014), 136-147.

[22] A. Perez Fornos, N. Guinand, R. van de Berg, R. Stokroos, S. Micera, H. Kingma, et al. Artificial balance: restoration of the vestibulo-ocular reflex in humans with a prototype vestibular neuroprosthesis. Frontiers in neurology 5 (2014), 66.

[23] A. Ramos de Miguel, J.C. Falcon Gonzalez and A. Ramos Macias, Vestibular Response to Electrical Stimulation of the Otolith Organs. Implications in the Development of A Vestibular Implant for the Improvement of the Sensation of Gravitoinertial Accelerations, J Int Adv Otol 13(2) (2017), 154-161.

[24] G.Y. Fridman and C.C. Della Santina, Progress toward development of a multichannel vestibular prosthesis for treatment of bilateral vestibular deficiency, Anatomical record 295(11) (2012), 2010-2029.

[25] J.T. Rubinstein, S. Bierer, C. Kaneko, L. Ling, K. Nie, T. Oxford, et al. Implantation of the semicircular canals with preservation of hearing and rotational sensitivity: a vestibular neurostimulator suitable for clinical research. Otology \& neurotology : official publication of the American Otological Society, American Neurotology Society [and] European Academy of Otology and Neurotology 33(5) (2012), 789-796.

[26] R. van de Berg, N. Guinand, M. Ranieri, S. Cavuscens, T.A. Khoa Nguyen, J.P. Guyot, et al. The Vestibular Implant Input Interacts with Residual Natural Function, Frontiers in neurology 8 (2017), 644.

[27] C. Phillips, L. Ling, T. Oxford, A. Nowack, K. Nie, J.T. Rubinstein, et al. Longitudinal performance of an implantable vestibular prosthesis, Hear Res 322 (2015), 200-211.

[28] R. van de Berg, N. Guinand, T.A. Nguyen, M. Ranieri, S. Cavuscens, J.P. Guyot, et al. The vestibular implant: frequency-dependency of the electrically evoked vestibulo- ocular reflex in humans, Frontiers in systems neuroscience 8 (2014), 255.

[29] P.J. Boutros, D.P. Schoo, M. Rahman, N.S. Valentin, M.R. Chow, A.L. Ayiotis, et al. Continuous Vestibular Implant Stimulation Partially Restores Eye-Stabilizing Reflexes, Journal of Clinical Investigation Insight. 2019.

[30] J.T. Rubinstein, L. Ling, A. Nowack, K. Nie and J.O. Phillips, Results From a Second-Generation Vestibular Implant in Human Subjects: Diagnosis May Impact Electrical Sensitivity of Vestibular Afferents. Otology \& neurotology: official publication of the American Otological Society, American Neurotology Society [and] European Academy of Otology and Neurotology 41(1) (2020), 68-77.

[31] A.P. Fornos, R. van de Berg, S. Armand, S. Cavuscens, M. Ranieri, C. Cretallaz, et al. Cervical myogenic potentials and controlled postural responses elicited by a prototype vestibular implant, Journal of neurology 266(Suppl 1) (2019), 33-41.

[32] C. Phillips, C. Defrancisci, L. Ling, K. Nie, A. Nowack, J.O. Phillips, et al. Postural responses to electrical stimulation of the vestibular end organs in human subjects, Experimental Brain Research 229(2) (2013), 181-195.

[33] N. Guinand, R. Van de Berg, S. Cavuscens, R. Stokroos, M. Ranieri, M. Pelizzone, et al. Restoring Visual Acuity in Dynamic Conditions with a Vestibular Implant, Front Neurosci 10 (2016), 577

[34] D. Starkov, N. Guinand, F. Lucieer, M. Ranieri, S. Cavuscens, M. Pleshkov, et al. Restoring the High-Frequency Dynamic Visual Acuity with a Vestibular Implant Prototype in Humans, Audiology \& neuro-otology 2019, 1-5.

[35] R. van de Berg, N. Guinand, R.J. Stokroos, J.P. Guyot and H. Kingma, The vestibular implant: quo vadis? Frontiers in neurology 2 (2011), 47.

[36] N. Guinand, R. van de Berg, S. Cavuscens, R.J. Stokroos, M. Ranieri, M. Pelizzone, et al. Vestibular Implants: 8 Years of Experience with Electrical Stimulation of the Vestibular Nerve in 11 Patients with Bilateral Vestibular Loss, ORL J Otorhinolaryngol Relat Spec 77(4) (2015), 227-240.

[37] Y. Agrawal, T. Bremova, O. Kremmyda and M. Strupp, Semicircular canal, saccular and utricular function in patients with bilateral vestibulopathy: analysis based on etiology, Journal of neurology 260(3) (2013), 876-883.

[38] B. Dobbels, G. Mertens, A. Gilles, A. Claes, J. Moyaert, R. van de Berg, et al. Cognitive Function in Acquired Bilateral Vestibulopathy: A Cross-Sectional Study on Cognition, Hearing, and Vestibular Loss, Front Neurosci 13 (2019), 340.

[39] S. Kim, Y.M. Oh, J.W. Koo and J.S. Kim, Bilateral vestibulopathy: clinical characteristics and diagnostic criteria. Otology \& neurotology: official publication of the American Otological Society, American Neurotology Society [and] European Academy of Otology and Neurotology 32(5) (2011), 812-817.

[40] A.A. Tarnutzer, C.J. Bockisch, E. Buffone and K.P. Weber, Hierarchical Cluster Analysis of Semicircular Canal and Otolith Deficits in Bilateral Vestibulopathy, Frontiers in neurology 9 (2018), 244.

[41] A.R. Bisdorff, J.P. Staab and D.E. Newman-Toker, Overview of the International Classification of Vestibular Disorders, Neurologic clinics 33(3) (2015), 541-550, vii.

[42] B. Dobbels, F. Lucieer, G. Mertens, A. Gilles, J. Moyaert, P. van de Heyning, et al. Vestibular testing and its relationship to symptoms and falls in 119 patients with bilateral vestibulopathy. 32nd Politzer Society Meeting; Warsaw, 2019. 
[43] D. Mayhew, V. Mendonca and B.V.S. Murthy, A review of ASA physical status - historical perspectives and modern developments, Anaesthesia 74(3) (2019), 373-379.

[44] A.L. Tamber, K.T. Wilhelmsen and L.I. Strand, Measurement properties of the Dizziness Handicap Inventory by cross-sectional and longitudinal designs, Health Qual Life Outcomes 7 (2009), 101.

[45] S.L. Whitney, D.M. Wrisley, K.E. Brown and J.M. Furman, Is perception of handicap related to functional performance in persons with vestibular dysfunction? Otology \& neurotology: official publication of the American Otological Society, American Neurotology Society [and] European Academy of Otology and Neurotology 25(2) (2004), 139-143.

[46] R. van de Berg, N. Guinand, J.P. Guyot, H. Kingma and R.J. Stokroos, The modified ampullar approach for vestibular implant surgery: feasibility and its first application in a human with a long-term vestibular loss, Frontiers in neurology 3 (2012), 18.

[47] G.C. Feigl, J.H. Fasel, F. Anderhuber, H. Ulz, R. Rienmuller, J.P. Guyot, et al. Superior vestibular neurectomy: a novel transmeatal approach for a denervation of the superior and lateral semicircular canals. Otology \& neurotology : official publication of the American Otological Society, American Neurotology Society [and] European Academy of Otology and Neurotology 30(5) (2009), 586-591.

[48] A.A. Tarnutzer, C.J. Bockisch, E. Buffone, S. Weiler, L.M. Bachmann and K.P. Weber, Disease-specific sparing of the anterior semicircular canals in bilateral vestibulopathy, Clinical neurophysiology: official journal of the International Federation of Clinical Neurophysiology 127(8) (2016), 2791-2801.

[49] G. Mantokoudis, Saber A.S. Tehrani, J.C. Kattah, K. Eibenberger, C.I. Guede, D.S. Zee, et al. Quantifying the vestibulo-ocular reflex with video-oculography: nature and frequency of artifacts, Audiology \& neuro-otology 20(1) (2015), 39-50.

[50] H.G. MacDougall, L.A. McGarvie, G.M. Halmagyi, S.J. Rogers, L. Manzari, A.M. Burgess, et al. A new saccadic indicator of peripheral vestibular function based on the video head impulse test, Neurology 87(4) (2016), 410-418.

[51] B.K. Ward, R. Van de Berg, V. Van Rompaey, A.R. Bisdorff, E. HT, M.S. Welgampola, et al. Barany Society ICVD Proposal Superior Semicircular Canal Dehiscence Syndrome (SCDS). in press.

[52] K.S. Noij, B.S. Herrmann, J.J. Guinan, Jr. and S.D. Rauch, Cervical Vestibular Evoked Myogenic Potentials in Meniere's Disease: A Comparison of Response Metrics. Otology \& neurotology: official publication of the American Otological Society, American Neurotology Society [and] European Academy of Otology and Neurotology 40(3) (2019), e215-e24.

[53] C. Fujimoto, T. Murofushi, K. Sugasawa, Y. Chihara, M. Ushio, T. Yamasoba, et al. Bilateral vestibulopathy with dissociated deficits in the superior and inferior vestibular systems, The Annals of otology, rhinology, and laryngology 121(6) (2012), 383-888.

[54] S.M. Rosengren, M.S. Welgampola and R.L. Taylor, Vestibular-Evoked Myogenic Potentials in Bilateral Vestibulopathy, Frontiers in neurology 9 (2018), 252.

[55] V.C. Zingler, E. Weintz, K. Jahn, K. Botzel, J. Wagner, D. Huppert, et al. Saccular function less affected than canal function in bilateral vestibulopathy, Journal of neurology 255(9) (2008), 1332-1336.

[56] B. Dobbels, F. Lucieer, G. Mertens, A. Gilles, J. Moyaert, P. van de Heyning, et al. Prospective cohort study on the predictors of fall risk in 119 patients with bilateral vestibulopathy, PloS one 15(3) (2020), e0228768.

[57] D.Q. Sun, M. Lehar, C. Dai, L. Swarthout, A.M. Lauer, J.P. Carey, et al. Histopathologic Changes of the Inner ear in Rhesus Monkeys After Intratympanic Gentamicin Injection and Vestibular Prosthesis Electrode Array Implantation, $J$ Assoc Res Otolaryngol 16(3) (2015), 373-387.

[58] T.C. Hain, M. Cherchi and D.A. Yacovino, Bilateral Vestibular Weakness, Frontiers in neurology 9 (2018), 344.

[59] C.D. Hall, S.J. Herdman, S.L. Whitney, S.P. Cass, R.A. Clendaniel, T.D. Fife, et al. Vestibular Rehabilitation for Peripheral Vestibular Hypofunction: An Evidence-Based Clinical Practice Guideline: From the American Physical Therapy Association Neurology Section, Journal of neurologic physical therapy: JNPT 40(2) (2016), 124-155.

[60] D.P. Schoo, P.J. Boutros, M.R. Chow, Y. Gimmon, S.P. Bowditch, M. Rahman, et al., editors. Multichannel Vestibular Implant Early Feasibility Study: Safety and Audiometric Outcomes. Barany Society; 2018; Uppsala: Journal of Vestibular Research.

[61] M.I. Kos, G. Feigl, F. Anderhuber, C. Wall, J.H. Fasel and J.P. Guyot, Transcanal approach to the singular nerve. Otology \& neurotology: official publication of the American Otological Society, American Neurotology Society [and] European Academy of Otology and Neurotology 27(4) (2006), 542-546. 